PROCEEDINGS OF THE

AMERICAN MATHEMATICAL SOCIETY

Volume 136, Number 7, July 2008, Pages 2493-2498

S 0002-9939(08)09378-7

Article electronically published on March 19, 2008

\title{
A NEW CHARACTERIZATION OF ULTRASPHERICAL POLYNOMIALS
}

\author{
R. LASSER AND J. OBERMAIER \\ (Communicated by Peter A. Clarkson)
}

\begin{abstract}
We characterize the class of ultraspherical polynomials in between all symmetric orthogonal polynomials on $[-1,1]$ via the special form of the representation of the derivatives $p_{n+1}^{\prime}(x)$ by $p_{k}(x), k=0, \ldots, n$.
\end{abstract}

Let $\left(p_{n}(x)\right)_{n \in \mathbb{N}_{0}}$ be a symmetric orthonormal polynomial sequence with respect to a symmetric probability measure $\pi$ with supp $\pi \subseteq[-1,1]$, that is, $p_{n}$ is an algebraic polynomial with $\operatorname{deg} p_{n}=n, \int p_{n} p_{k} d \pi=\delta_{n, k}$ and $p_{n}$ has a positive leading coefficient. It is well-known [2] that the $p_{n}(x)$ satisfy a recursion formula

$$
x p_{n}(x)=A_{n+1} p_{n+1}(x)+A_{n} p_{n-1}(x) \quad \text { for } n \in \mathbb{N}_{0},
$$

where $p_{-1} \equiv 0, \quad p_{0}(x)=1$, and $A_{n}>0, n \geq 1$ are bounded. Since supp $\pi \subseteq[-1,1]$ the $n$ simple zeroes of $p_{n}(x)$ are contained in $]-1,1\left[\right.$. Hence $p_{n}(1) \neq 0$ for each $n \in \mathbb{N}_{0}$.

Conversely, by Favard's theorem [2] we know that a sequence of polynomials which fulfills (11) is an orthogonal polynomial sequence with respect to a symmetric measure on $\mathbb{R}$. Hence Favard's theorem is a characterization theorem for orthogonal polynomials. It is of great interest to characterize more special classes of orthogonal polynomials. A survey of such characterization theorems is given in [1]. One problem mentioned in 1 is to find all orthogonal polynomial sequences whose derivatives are special linear combinations of polynomials of the same system.

The purpose of this note keeps going in the same direction, namely to characterize the ultraspherical polynomials in between the class of symmetric orthonormal polynomials on $[-1,1]$ by means of the linear representation of $p_{n+1}^{\prime}(x)$ by $p_{k}(x), \quad k=0, \ldots, n$.

We prefer to use another normalization of the polynomials $p_{n}(x)$. Let

$$
R_{n}(x)=p_{n}(x) / p_{n}(1) .
$$

Then the recursion formula for $R_{n}(x)$ is

$$
x R_{n}(x)=a_{n} R_{n+1}(x)+c_{n} R_{n-1}(x) \quad \text { for } n \in \mathbb{N}_{0},
$$

where $R_{-1} \equiv 0, R_{0}(x)=1$ and $a_{0}=1$.

Since $R_{n+1}(1)=1$ yields $\lim _{x \rightarrow \infty} R_{n+1}(x)=\infty$ the leading coefficient of $R_{n+1}$ has to be positive, that is, $a_{n}>0$ for all $n \geq 1$. Additionally $R_{n}(1)=1$ implies

Received by the editors March 6, 2007.

2000 Mathematics Subject Classification. Primary 33C45; Secondary 42C05.

Key words and phrases. Orthogonal polynomials, ultraspherical polynomials.

(C)2008 American Mathematical Society Reverts to public domain 28 years from publication 
$a_{n}+c_{n}=1$ for all $n \geq 1$. From the theory of orthogonal polynomials [2] it is known that $c_{n} a_{n-1}>0$, which implies $c_{n}>0$ for $n \geq 1$. Hence it holds that $0<a_{n}, c_{n}<1$ for $n \geq 1$.

The coefficients of (11) and (2) are related by

$$
A_{n}=\sqrt{c_{n} a_{n-1}} \quad \text { for } n \in \mathbb{N} .
$$

Furthermore, $p_{n}(1)=\sqrt{h(n)}$, where $h(0)=1$ and

$$
h(n+1) c_{n+1}=a_{n} h(n) \quad \text { for } n \in \mathbb{N}_{0} .
$$

The ultraspherical polynomials $R_{n}^{(\alpha)}(x), \alpha>-1$ (normalized such that $R_{n}^{(\alpha)}(1)$ =1) satisfy

$$
\int_{-1}^{1} R_{n}^{(\alpha)}(x) R_{k}^{(\alpha)}(x) d \pi(x)=\delta_{n, k} \frac{1}{h(n)},
$$

where $d \pi(x)=c_{\alpha}\left(1-x^{2}\right)^{\alpha} d x, \quad c_{\alpha}=\frac{\Gamma(2 \alpha+2)}{2^{2 \alpha+1}(\Gamma(\alpha+1))^{2}}$. The recurrence coefficients are

$$
a_{n}=\frac{n+2 \alpha+1}{2 n+2 \alpha+1}, \quad c_{n}=\frac{n}{2 n+2 \alpha+1}, \quad n \in \mathbb{N} .
$$

The derivatives $R_{n+1}^{\prime}(x)$ (and $p_{n+1}^{\prime}(x)$ ) are polynomials of degree $n$. Hence

$$
R_{n+1}^{\prime}(x)=\sum_{k=0}^{n} d_{n, k} R_{k}(x)
$$

with linearization coefficients $d_{n, k}$. From [5, (7.32.5)] we obtain for ultraspherical polynomials $\left(R_{n}^{(\alpha)}\right)^{\prime}(x)=\frac{n(n+2 \alpha+1)}{2+2 \alpha} R_{n-1}^{(\alpha+1)}(x)$. In that case we can calculate $d_{n, k}$ from the so-called connection coefficients which connect $R_{n}^{(\alpha+1)}(x)$ and $R_{k}^{(\alpha)}(x)$; see 3. (9.1.2)]. We shall show that for $m \in \mathbb{N}_{0}$

$$
\begin{aligned}
& \left(R_{2 m+1}^{(\alpha)}\right)^{\prime}(x)=\gamma_{2 m+1} \sum_{k=0}^{m} R_{2 k}^{(\alpha)}(x) h(2 k) \quad \text { and } \\
& \left(R_{2 m+2}^{(\alpha)}\right)^{\prime}(x)=\gamma_{2 m+2} \sum_{k=0}^{m} R_{2 k+1}^{(\alpha)}(x) h(2 k+1),
\end{aligned}
$$

where $\gamma_{n}=\frac{(n+2 \alpha+1) n !}{(2+2 \alpha)(2 \alpha+3)_{n-1}}, \quad n \in \mathbb{N}$. More important, we shall prove that this special form of the linearization coefficients $d_{n, k}$ in (5) and (6) characterizes the ultraspherical polynomials in between the symmetric orthogonal polynomials on $[-1,1]$. For the orthonormal versions the formula (5) and (6) write as

$$
\begin{aligned}
& \left(p_{2 m+1}^{(\alpha)}\right)^{\prime}(x)=p_{2 m+1}^{(\alpha)}(1) \gamma_{2 m+1} \sum_{k=0}^{m} p_{2 k}^{(\alpha)}(x) p_{2 k}^{(\alpha)}(1) \quad \text { and } \\
& \left(p_{2 m+2}^{(\alpha)}\right)^{\prime}(x)=p_{2 m+2}^{(\alpha)}(1) \gamma_{2 m+2} \sum_{k=0}^{m} p_{2 k+1}^{(\alpha)}(x) p_{2 k+1}^{(\alpha)}(1) .
\end{aligned}
$$

Hence, (7) and (8) characterize the ultraspherical polynomials in between the symmetric orthonormal polynomials on $[-1,1]$. 
In order to formalize the calculation denote by $c_{00}$ the linear space of finite sequences and by

$$
\hat{f}(x)=\sum_{k=0}^{\infty} f(k) R_{k}(x) h(k)=\sum_{k=0}^{\infty} f(k) p_{k}(x) p_{k}(1)
$$

the Fourier expansion of $f \in c_{00}$. Further put $\epsilon_{n} \in c_{00}$ with

$$
\epsilon_{n}(k)=\frac{1}{h(k)} \delta_{n, k} \quad \text { for } k \in \mathbb{N}_{0},
$$

and define the convolution $\epsilon_{1} * f$ by

$$
\epsilon_{1} * f(k)=a_{k} f(k+1)+c_{k} f(k-1) \quad \text { for } k \in \mathbb{N}_{0},
$$

where we set $c_{0}=0$. The notion of convolution is motivated by the theory of polynomial hypergroups; compare 44. Of course, here we do not suppose that the orthogonal polynomials $R_{n}(x)$ induce a polynomial hypergroup.

Define recursively $\kappa_{n} \in c_{00}$ by $\kappa_{0} \equiv 0, \kappa_{1}=\epsilon_{0}$ and

$$
\kappa_{n+1}=\frac{1}{a_{n}}\left(\epsilon_{n}+\epsilon_{1} * \kappa_{n}-c_{n} \kappa_{n-1}\right) \quad \text { for } n \in \mathbb{N} .
$$

It is easy to check that supp $\kappa_{n} \subseteq\{0, \ldots, n\}$. Furthermore, most importantly it holds that

$$
\hat{\epsilon}_{n}(x)=R_{n}(x) \quad \text { and } \quad \hat{\kappa}_{n}(x)=R_{n}^{\prime}(x) \quad \text { for } n \in \mathbb{N}_{0} .
$$

To verify the latter identity first notify that by (3) we have

$$
\begin{aligned}
x \hat{f}(x) & =\sum_{k=0}^{\infty} f(k) x R_{k}(x) h(k) \\
& =\sum_{k=0}^{\infty} f(k)\left(a_{k} R_{k+1}(x)+c_{k} R_{k-1}(x)\right) h(k) \\
& =\sum_{k=1}^{\infty} c_{k} f(k-1) R_{k}(x) h_{k}+\sum_{k=0}^{\infty} a_{k} f(k+1) R_{k}(x) h_{k} \\
& =\left(\epsilon_{1} * f\right)^{\wedge}(x)
\end{aligned}
$$

for $f \in c_{00}$. Differentiating the recursion formula (2) yields

$$
R_{n+1}^{\prime}(x)=\frac{1}{a_{n}}\left(R_{n}(x)+x R_{n}^{\prime}(x)-c_{n} R_{n-1}^{\prime}(x)\right) .
$$

Therefore the second identity in (10) easily follows by induction.

Lemma 1. Let $\left(R_{n}(x)\right)_{n \in \mathbb{N}_{0}}$ be an orthogonal polynomial sequence defined by (2) with $a_{n}>0$ and $a_{n}+c_{n}=1$ for all $n \in \mathbb{N}$. If the recursion coefficients $c_{n}$ satisfy

$$
c_{n+1}=\frac{(n+1) c_{n}}{2 c_{n}+n} \quad \text { for } n \in \mathbb{N},
$$

then $\left(R_{n}(x)\right)_{n \in \mathbb{N}_{0}}$ belongs to the class of ultraspherical polynomials. More precisely $R_{n}(x)=R_{n}^{(\alpha)}(x)$, where $\alpha=\frac{1}{2}\left(\frac{1}{c_{1}}-3\right)$. Conversely, the recursion coefficients $c_{n}$ of $R_{n}^{(\alpha)}(x)$ satisfy (11) with $c_{1}=\frac{1}{2 \alpha+3}$. 
Proof. Beginning with $c_{1}=\frac{1}{2 \alpha+3}$ a simple induction shows that the $c_{n}$ defined by (11) yield exactly the $c_{n}$ of (4). Conversely, for the coefficients $c_{n}$ of (4) the identity (11) holds true.

The next theorem is the main result and gives a characterization of ultraspherical polynomials.

Theorem 1. Let $\left(R_{n}(x)\right)_{n \in \mathbb{N}_{0}}$ be an orthogonal polynomial sequence defined by (2) with $a_{n}>0$ and $a_{n}+c_{n}=1$ for all $n \in \mathbb{N}$, and $h(n)$ defined by (3). The following three conditions are equivalent:

(i) It holds that

$$
\begin{aligned}
& R_{2 m+1}^{\prime}(x)=\sigma_{2 m+1} \sum_{j=0}^{m} R_{2 j}(x) h(2 j) \quad \text { and } \\
& R_{2 m+2}^{\prime}(x)=\sigma_{2 m+2} \sum_{j=0}^{m} R_{2 j+1}(x) h(2 j+1) \quad \text { for } m \in \mathbb{N}_{0},
\end{aligned}
$$

where $\sigma_{2 m+1}$ and $\sigma_{2 m+2}$ are constants.

(ii) $c_{n+1}=\frac{(n+1) c_{n}}{2 c_{n}+n} \quad$ for all $n \in \mathbb{N}$.

(iii) $\left(R_{n}(x)\right)_{n \in \mathbb{N}_{0}}$ belongs to the class of ultraspherical polynomials.

The constants in (i) are given by $\sigma_{n+1}=\frac{n+1}{a_{n} h(n)}$ for all $n \in \mathbb{N}_{0}$.

Proof. By (10) we have $\sigma_{n+1}=\kappa_{n+1}(n)$ for all $n \in \mathbb{N}_{0}$. Moreover, we easily derive

$$
\kappa_{n+1}(n)=\frac{1}{a_{n}}\left(\frac{1}{h(n)}+c_{n} \kappa_{n}(n-1)\right) \quad \text { for } n \in \mathbb{N} .
$$

Applying induction this identity implies

$$
\sigma_{n+1}=\kappa_{n+1}(n)=\frac{n+1}{a_{n} h(n)} \quad \text { for } n \in \mathbb{N}_{0} .
$$

In fact, $\kappa_{1}(0)=1=\frac{1}{a_{0} h(0)}$, and supposing $\kappa_{n}(n-1)=\frac{n}{a_{n-1} h(n-1)}=\frac{n}{c_{n} h(n)}$, we obtain

$$
\kappa_{n+1}(n)=\frac{1}{a_{n}}\left(\frac{1}{h(n)}+\frac{n}{h(n)}\right)=\frac{n+1}{a_{n} h(n)} .
$$

We first prove (i) $\Rightarrow$ (ii).

Let $n \geq 2$. Taking into account (91) and (10) the assumption made in (i) implies

$$
\begin{aligned}
\sigma_{n+1}=\kappa_{n+1}(n) & =\kappa_{n+1}(n-2) \\
& =\frac{\epsilon_{n}(n-2)+c_{n-2} \kappa_{n}(n-3)+a_{n-2} \kappa_{n}(n-1)-c_{n} \kappa_{n-1}(n-2)}{a_{n}} \\
& =\frac{\left(c_{n-2}+a_{n-2}\right) \kappa_{n}(n-1)-c_{n} \kappa_{n-1}(n-2)}{a_{n}}=\frac{\sigma_{n}-c_{n} \sigma_{n-1}}{a_{n}} .
\end{aligned}
$$

Hence, by (12) and (3) we get

$$
n+1=h(n)\left(\frac{n}{c_{n} h(n)}-\frac{c_{n}(n-1)}{c_{n-1} h(n-1)}\right)=\frac{n}{c_{n}}-\frac{(n-1) a_{n-1}}{c_{n-1}},
$$

which gives

$$
c_{n}=\frac{n c_{n-1}}{2 c_{n-1}+(n-1)} \quad \text { for } n \geq 2 .
$$

The implication (ii) $\Rightarrow$ (iii) follows by Lemma 1 , 
Suppose that $R_{n}(x)=R_{n}^{(\alpha)}(x), \alpha>-1$. If we show that

$$
\begin{aligned}
& \kappa_{2 m+1}(2 j)=\frac{2 m+1}{a_{2 m} h(2 m)}, \quad \kappa_{2 m+1}(2 j+1)=0 \quad \text { and } \\
& \kappa_{2 m+2}(2 j)=0, \quad \kappa_{2 m+2}(2 j+1)=\frac{2 m+2}{a_{2 m+1} h(2 m+1)} \quad \text { for } j=0, \ldots, m,
\end{aligned}
$$

then (iii) $\Rightarrow$ (ii) holds. Especially $\sigma_{n+1}=\frac{n+1}{a_{n} h(n)}=\frac{(n+2 \alpha+2)(n+1) !}{(2+2 \alpha)(2 \alpha+3)_{n}}$.

To prove (13) we use induction on $m$. The calculations are based on (9).

We start with $\kappa_{1}(0)=1, \kappa_{2}(0)=0, \kappa_{2}(1)=\frac{2}{a_{1} h(1)}$. Further, using (11) we get

$$
\begin{aligned}
\kappa_{3}(0) & =\frac{1}{a_{2}}\left(\kappa_{2}(1)-c_{2}\right)=\frac{2 c_{1}-a_{1} c_{2}}{a_{1} a_{2}} \\
& =\frac{2 c_{2} c_{1}+c_{2}-a_{1} c_{2}}{a_{1} a_{2}}=\frac{3 c_{1} c_{2}}{a_{1} a_{2}}=\frac{3}{a_{2} h(2)}, \\
\kappa_{3}(1) & =0, \quad \kappa_{3}(2)=\frac{3}{a_{2} h(2)} .
\end{aligned}
$$

Finally, $\kappa_{3}(2)=\kappa_{3}(0)$ and (11) yield

$$
\begin{aligned}
& \kappa_{4}(0)=0, \kappa_{4}(1)=\frac{1}{a_{3}}\left(\kappa_{3}(2)-c_{3} \kappa_{2}(1)\right)=\frac{4 c_{1} c_{2} c_{3}}{a_{1} a_{2} a_{3}}=\frac{4}{a_{3} h(3)}, \\
& \kappa_{4}(2)=0, \quad \kappa_{4}(3)=\frac{4}{a_{3} h(3)} .
\end{aligned}
$$

Now suppose that (13) holds for $m-1$. It follows by (11)

$$
\begin{aligned}
\kappa_{2 m+1}(0) & =\frac{\kappa_{2 m}(1)-c_{2 m} \kappa_{2 m-1}(0)}{a_{2 m}} \\
& =\frac{2 m}{a_{2 m} a_{2 m-1} h(2 m-1)}-\frac{c_{2 m}(2 m-1)}{a_{2 m} a_{2 m-2} h(2 m-2)} \\
& =\frac{2 m}{a_{2 m} c_{2 m} h(2 m)}-\frac{c_{2 m} a_{2 m-1}(2 m-1)}{a_{2 m} c_{2 m-1} c_{2 m} h(2 m)} \\
& =\frac{1}{a_{2 m} h(2 m)} \frac{2 m c_{2 m-1}-c_{2 m}\left(1-c_{2 m-1}\right)(2 m-1)}{c_{2 m} c_{2 m-1}}=\frac{2 m+1}{a_{2 m} h(2 m)}
\end{aligned}
$$

and therefore

$$
\begin{aligned}
\kappa_{2 m+1}(2 j) & =\frac{a_{2 j} \kappa_{2 m}(2 j+1)+c_{2 j} \kappa_{2 m}(2 j-1)-c_{2 m} \kappa_{2 m-1}(2 j)}{a_{2 m}} \\
& =\frac{\kappa_{2 m}(1)-c_{2 m} \kappa_{2 m-1}(0)}{a_{2 m}}=\frac{2 m+1}{a_{2 m} h(2 m)} \quad \text { for } j=1, \ldots, m-1 .
\end{aligned}
$$

Finally

$$
\begin{aligned}
\kappa_{2 m+1}(2 m) & =\frac{1}{a_{2 m} h(2 m)}+\frac{c_{2 m} \kappa_{2 m}(2 m-1)}{a_{2 m}} \\
& =\frac{1}{a_{2 m} h(2 m)}+\frac{c_{2 m} 2 m}{a_{2 m} a_{2 m-1} h(2 m-1)}=\frac{2 m+1}{a_{2 m} h(2 m)} .
\end{aligned}
$$

Furthermore,

$$
\begin{aligned}
\kappa_{2 m+1}(2 j+1) & =\frac{a_{2 j+1} \kappa_{2 m}(2 j+2)+c_{2 j+1} \kappa_{2 m}(2 j)-c_{2 m} \kappa_{2 m-1}(2 j+1)}{a_{2 m}} \\
& =0 \quad \text { for } j=0, \ldots, m-1 .
\end{aligned}
$$


Just in the same way one derives from the induction hypothesis

$$
\begin{aligned}
\kappa_{2 m+2}(2 j+1) & =\frac{2 m+2}{a_{2 m+1} h(2 m+1)} \\
\kappa_{2 m+2}(2 j) & =0 \quad \text { for } j=0, \ldots, m .
\end{aligned}
$$

Finally, using the relationship between (11) and (2) discussed at the beginning we get the following corollary.

Corollary 1. Let $\left(p_{n}(x)\right)_{n \in \mathbb{N}_{0}}$ be a symmetric orthonormal polynomial sequence with respect to a symmetric probability measure with supp $\pi \subseteq[-1,1]$. Then the following two conditions are equivalent:

(i)

$$
\begin{aligned}
& p_{2 m+1}^{\prime}(x)=\sigma_{2 m+1} p_{2 m+1}(1) \sum_{k=0}^{m} p_{2 k}(x) p_{2 k}(1), \\
& p_{2 m+2}^{\prime}(x)=\sigma_{2 m+2} p_{2 m+2}(1) \sum_{k=0}^{m} p_{2 k+1}(x) p_{2 k+1}(1), \quad m \in \mathbb{N}_{0}, \\
& \text { where } \sigma_{2 m+1} \text { and } \sigma_{2 m+2} \text { are constants }
\end{aligned}
$$

(ii) $\left(p_{n}(x)\right)_{n \in \mathbb{N}_{0}}$ belongs to the class of ultraspherical polynomials.

The constants in (i) are given by $\sigma_{n+1}=\frac{n+1}{a_{n} h(n)}$ for all $n \in \mathbb{N}_{0}$.

\section{REFERENCES}

[1] W.A. Al-Salam, Characterization theorems for orthogonal polynomials, in: P. Nevai (Ed.), Orthogonal Polynomials, 1-24, NATO Adv. Sci. Inst. Ser. C Math. Phys. Sci., 294, Kluwer Acad. Publ., Dordrecht, 1990. MR.1100286 (92g:42011)

[2] T.S. Chihara, An Introduction to Orthogonal Polynomials, Gordon and Breach, New York, 1978. MR0481884 (58:1979)

[3] M.E.H. Ismail, Classical and Quantum Orthogonal Polynomials in One Variable, Cambridge University Press, Cambridge, 2005. MR2191786 (2007f:33001)

[4] R. Lasser, Orthogonal polynomials and hypergroups II - The symmetric case, Trans. Amer. Math. Soc. 341 (1994), 749-770. MR.1139495(94d:33005)

[5] G. Szegö, Orthogonal Polynomials, Amer. Math. Soc., Providence, RI, 1959. MR0106295 $(21: 5029)$

Helmholtz Zentrum München, German Research Center for Environmental Health, Institute of Biomathematics And Biometry, Ingolstädter Landstrasse 1, 85764 Neuherberg, Germany; and Münich University of Technology, Centre of Mathematics, 85748 GARChing, GERMANY

E-mail address: lasser@helmholtz-muenchen.de

Helmholtz Zentrum München, German Research Center for Environmental Health, Institute of Biomathematics and Biometry, Ingolstädter Landstrasse 1, 85764 NeuherBerg, Germany

E-mail address: josef.obermaier@helmholtz-muenchen.de 